\title{
Effects of ultrasonicated methylcellulose coating on French fries during deep frying
}

\begin{abstract}
Ultrasonic treatment (UT) effects on methylcellulose (MC) for the food coating purpose prior to deep-fat frying process of potato strips were demonstrated. Different concentration of MC (0.5$2.0 \mathrm{wt} / \mathrm{vol} \%$ ) solutions were subjected to UT at $20 \mathrm{~W}$ to evaluate the effect of UT treated coatings on rheological behavior of the coated samples and their efficiency in minimizing the oil uptake. Application of UT to methylcellulose (UTMC) clearly showed a phase transition from fluid- to gel-like between 20 and $65^{\circ} \mathrm{C}$ with the increasing in the oscillatory frequency and temperature ramp test of UTMC showed the sol-gel transition occurrence is lower than non-UTMC at 20 and $29^{\circ} \mathrm{C}$, respectively, within the linear viscoelastic region. When the MC concentrations were varied, the most effective concentration was noticed to occur at 1.0 (wt/vol\%) UTMC whereby the relative variation of water retention and oil uptake were reduced to about 49.9 and $31.0 \%$, respectively, as compared to $1.0 \mathrm{wt} / \mathrm{vol} \%$ non-UTMC formulation. Experimental results affirm that UTMC coating application can be used successively to improve rheological properties of MC by minimizing oil uptake on fried food.
\end{abstract}

